\title{
Hear! Hear! Against alLODDS, we might have a LiNeaR predictive model for prognosis of non-small cell lung cancer from $\mathbf{p N}+$ nodal status
}

\author{
Gaetano Rocco, MD, FRCSEd
}

\footnotetext{
From the Division of Thoracic Surgery, Department of Thoracic Surgical and Medical Oncology, Istituto Nazionale Tumori, IRCCS, Pascale Foundation, Naples, Italy.

Disclosures: Author has nothing to disclose with regard to commercial support.

Received for publication Dec 1, 2016; accepted for publication Dec 6, 2016; available ahead of print Jan 12, 2017. Address for reprints: Gaetano Rocco, MD, FRCSEd, Division of Thoracic Surgery, Department of Thoracic Surgical and Medical Oncology, Istituto Nazionale Tumori, IRCCS, Pascale Foundation, Via Semmola 81, 80131, Naples, Italy (E-mail: g.rocco@istitutotumori.na.it).

J Thorac Cardiovasc Surg 2017;153:710 $0022-5223 / \$ 36.00$

Copyright (c) 2016 by The American Association for Thoracic Surgery http://dx.doi.org/10.1016/j.jtcvs.2016.12.005
}

Metastatic non-small cell lung cancer to the mediastinal nodal compartment is considered to be an ominous sign of disease dissemination. Although stage N3 is unanimously accepted as an indication for definitive chemoradiotherapy, several factors argue against a precise delineation of the prognostic profile of stage $\mathrm{N} 1$ or $\mathrm{N} 2$ disease, such as the skip phenomenon and the acceptable survival rates after primary surgery for single station N2 involvement. ${ }^{1}$ In addition, the value of the positron emission tomography standard uptake value in predicting nodal disease and the attendant prognostic outlook remains controversial. Under the circumstances, an aggressive preoperative mediastinal exploration of positron emission tomography-positive nodes is usually advocated to rule out primary surgery, albeit according to a recent meta-analysis of prospective, randomized trials of bi- or trimodality regimens for locally advanced non-small cell lung cancer, the surgical arms seem to portend equal or even better prognosis compared with medical treatment. Zhao and colleagues ${ }^{3}$ have taken this line of reasoning further by proposing a prognostic scoring system of nodal involvement from lung cancer. They studied 1097 patients with resectable adenocarcinoma who did not undergo preresection mediastinal nodal staging nor receive induction therapy. Intraoperatively, a radical (the authors use "skeletonize" to signify the extent of nodal dissection) lymph node dissection was performed. At final pathology, lymph node ratio (LNR) and log odds ratio (LODDS) were calculated and combined with the standard $\mathrm{pN}+$ status to stratify surgical patients into predictive categories of overall and recurrence-free survival. The strengths of this study reside both in the attempt at reducing arbitrariness in selecting nodal positivity thresholds (with the recursive partitioning method) and in using parameters that are readily available in the pathology reports. In a time when biomolecular markers are increasingly used to help predict prognosis, the $\mathrm{pN}+/ \mathrm{LNR} / \mathrm{LODDS}$ model may help identify those patients at risk for early recurrence. This information could then be used to modify follow-up schemes or direct decisions

\section{References}

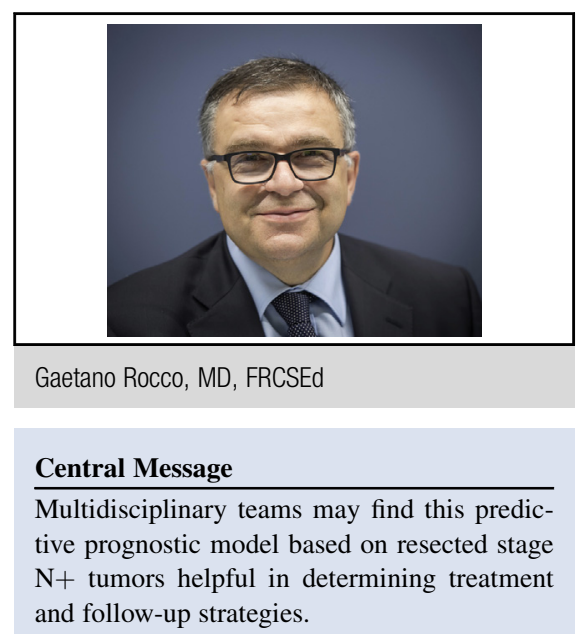

See Article page 702.

regarding adjuvant treatments. Also, the work by Zhao and colleagues ${ }^{3}$ supports performing radical mediastinal lymphadenectomy to obtain the information necessary to construct the $\mathrm{pN}+/ \mathrm{LNR} / \mathrm{LODDS}$ model. In agreement with an approach already advocated by several European surgeons, this study suggests that as long as the lung adenocarcinoma is resectable (ie, no stage $\mathrm{N} 3$ and no bulky stage $\mathrm{N} 2$ nodes), it should be subjected to primary surgery entailing a complete mediastinal nodal dissection to implement the $\mathrm{pN}+/ \mathrm{LNR} /$ LODDS model. The results of this study should be externally validated before uniform adoption in clinical practice as a predictive model. Nevertheless, Zhao and colleagues emphasize the need to combine a carefully tailored pulmonary resection with a meticulous mediastinal lymphadenectomy, either via an open or minimally invasive approach. The pN/LNR/LODDS model may serve the purpose to help standardize surgical treatment. This is worth a try.

1. Rocco G, Nason K, Brunelli A, Varela G, Waddell T, Jones DR. Management of stage IIIA (N2) non-small cell lung cancer: a transatlantic perspective. J Thorac Cardiovasc Surg. 2016;151:1235-8.

2. McElnay PJ, Choong A, Jordan E, Song F, Lim E. Outcome of surgery versus radiotherapy after induction treatment in patients with N2 disease: systematic review and meta-analysis of randomised trials. Thorax. 2015;70:764-8.

3. Zhao Y, Li G, Zheng D, Jia M, Dai W, Sun Y, et al. The prognostic value of lymph node ratio (LNR) and log odds of positive lymph nodes (LODDS) in patients with lung adenocarcinoma. J Thorac Cardiovasc Surg. 2017;153:702-9.

4. Lim E, McElnay PJ, Rocco G, Brunelli A, Massard G, Toker A, et al. Invasive mediastinal staging is irrelevant for PET/CT positive N2 lung cancer if the primary tumour and ipsilateral lymph nodes are resectable. Lancet Respir Med. 2015;3:e32-3. 